\title{
Low-Complexity Wireless Monitoring of Respiratory Movements Using Ultra-Wideband Impulse Response
}

\author{
Estimation
}

Furrukh Sana, Tarig Ballal, Tareq Y. Al-Naffouri, and Ibrahim Hoteit

\begin{abstract}
In this paper; we present a comprehensive scheme for wireless monitoring of the respiratory movements in humans. Our scheme overcomes the challenges low signal-tonoise ratio, background clutter and high sampling rates. It is based on the estimation of the ultra-wideband channel impulse response. We suggest techniques for dealing with background clutter in situations when it might be time variant. We also present a novel methodology for reducing the required sampling rate of the system significantly while achieving the accuracy offered by the Nyquist rate. Performance results from simulations conducted with pre-recorded respiratory signals demonstrate the robustness of our scheme for tackling the above challenges and providing a low-complexity solution for the monitoring of respiratory movements.
\end{abstract}

\section{Introduction}

Respiratory parameters such as respiratory rate and amplitude provide vital information about a person's state of health. Diagnosis of several illnesses and disorders, such as the sleep apnea, is based on the analysis of recordings of respiratory movements while the patient is asleep [1]. Such recordings are usually performed overnight in isolated hospital environments using polysomnography during which the patient is required to sleep under conditions of restricted motion while connected to numerous devices and electrodes [2]. Furthermore, to assess the effectiveness of treatment, the study might be repeated several 
times. The associated costs and discomfort can be high for the patient. As such, a noninvasive non-contact technique that offers low cost and reliable monitoring of respiratory movements is needed.

Ultra-wideband (UWB) technology offers the possibility of monitoring respiratory movements non-invasively and wirelessly. Indeed, UWB technology has been increasingly studied for ranging and imaging applications in medical environments. Compared with narrowband technologies, UWB offers the large bandwidth suitable for high-resolution ranging while operating in a low-power regime [3]. UWB signals create no or minimal interference with other sensitive equipment in the surroundings, which is of critical importance in medical environments. Although, applications such as the monitoring of respiratory movements and diagnonis of the sleep apnea have been considered in the past, they have been limited by several practical challenges. Most earlier studies, such as [4], [5] and [6], focus on estimating only vital signs, such as breathing and heart rates. Diagnosis of many illnesses and disorders, however, requires continous monitoring of the respiratory amplitude to detect abnormalities in the breathing pattern. This requires accurate tracking of respiratory signal with high range resolution.

Monitoring via UWB poses several challenges including multipath effects, low signalto-noise ratios (SNR) and the requirement of high sampling rates. These challenges are compounded by non-isolated and possibly time-variant environments. Solution for some of these issues can be found in the literature but, to the best of our knowledge, no comprehensive scheme has been suggested that simultaneously deals with all of these issues. Many earlier techniques were based on time-of-arrival (TOA) methods that rely on accurate identification of the direct path component. However, in a multipath environment, a direct path may not exist or it may not be the strongest [8]. Some schemes, such as the one presented in [9], assume that the multipath environment is known and time-invariant to deal with this issue, but this assumption is not practical in any non-isolated environment. Other schemes, such as those presented in [10,11], attempt to identify the direct path, which is a challenging task and adds additional complexity to the problem.

To improve the effective SNR, a technique was suggested in [7] that utilizes the redundant information available in the sub-peaks of the received signal. The effectiveness of this 
technique is, however, limited. A more widely used technique in typical UWB systems is to utilize multiple pulse transmissions to build a stronger received signal profile through averaging $[4,5]$. Because of their large bandwidths, UWB systems require high sampling rates to recover information accurately from the received signals. A solution to this problem has not been considered in the literature. Almost all techniques rely on equipment such as the digital oscilloscope as their front-end hardware to achieve good tracking accuracy $[7,9]$. Dependence on such complex hardware is a major bottleneck in any efficient and cost-effective UWB solution.

In this paper, we present a low-complexity UWB technique for monitoring respiratory movements of a human subject. Our goal here is to develop a low-complexity solution that provides reliable tracking performance in a realistic environment. We suggest a simple and novel technique to mitigate the effects of multipaths in a time varying environment. Unlike UWB schemes proposed previously in the literature, our technique reduces the required sampling rate significantly, which not only lowers the complexity of the proposed scheme but also eases the hardware requirements. We formulate the problem from a sparse signal estimation perspective using a Bayesian framework, which helps us in achieving good tracking performance against low SNR values. Simulation results demonstrate the robustness of our scheme in providing solutions to be above-mentioned challenges while maintaining low levels of complexity.

The remainder of this paper is organized as follows. Section 2 provides a brief overview of ultra-wideband technology and discusses localization issues in the presence of noise and clutter. Section 3 defines the signal model for the transmitted and received signals and describes the relationship between the received measurements and the sparse vector whose evolutions represent respiratory movements. A brief overview of the Support Agnostic Bayesian Matching Pursuit (SABMP) algorithm, used for sparse signal recovery, is presented in Section 4. We summarize our scheme in Section 5. Section 6 presents the simulation results and performance analysis for the tracking of a pre-recorded respiratory signal. The paper concludes with a brief summary of the proposed approach, its novelty and the achieved performance, in Section 7. 


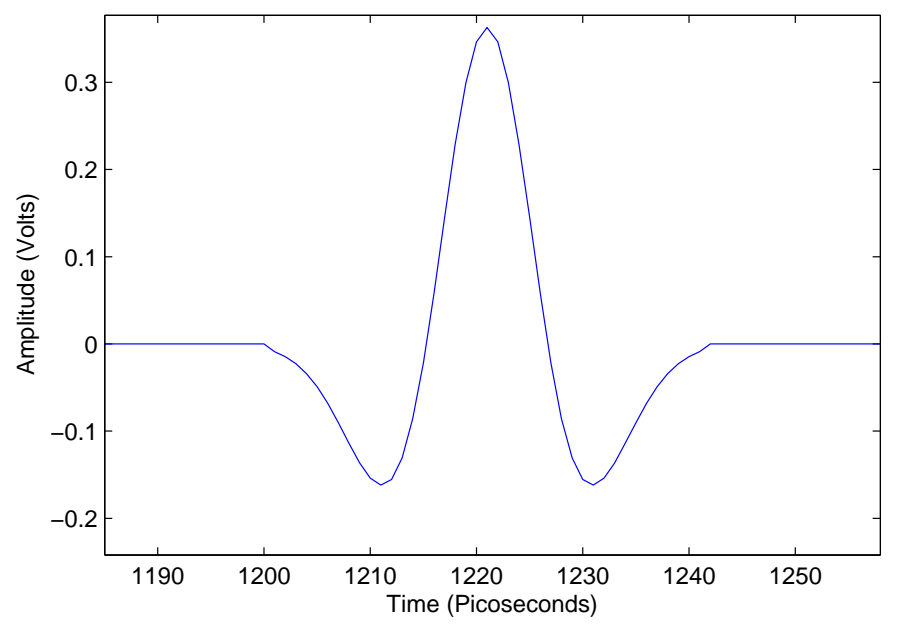

Figure 1: An ultra-wideband pulse

\section{Ultra-Wideband Systems}

UWB systems essentially use nano- or picosecond pulses as their transmitted signals. Figure 1 shows the typical shape of a transmitted pulse in a UWB system. The pulse in the figure is a second derivative Gaussian pulse with its mathematical expression given by [12]:

$$
p_{t x}(t)=\frac{1-\left((t-\mu) / \sigma^{2}\right)}{\sqrt{2 \pi} \sigma} \exp \left(-(t-\mu) / 2 \sigma^{2}\right)
$$

where $\mu=T_{w} / 2, \sigma=T_{w} / 7$ and $T_{w}$ is the pulse width.

As mentioned in Section 1, despite its advantages, wireless monitoring through UWB poses several practical challenges. These challenges are discussed in the following subsections.

\subsection{Signal-to-Noise Ratio}

Because of their low power profiles under FCC regulations [16], typical UWB systems cannot be expected to operate in regimes with high signal-to-noise ratios (SNR). A typical technique to improve the effective SNR is to transmit multiple pulses within a single measurement time window and average the received signals over that period [5]. Performance in low SNR regimes, however, can also be improved by exploiting the sparsity inherent in the UWB channel impulse response and through its estimation using a Bayesian framework. 
Section 3.3 presents details on how to formulate the respiratory movement tracking problem using UWB signals as a sparse signal estimation problem. We note that a multiple pulse transmission scheme can still prove to be useful for relaxing the sampling rate constraint as discussed further in the next section.

\subsection{Sampling Rates}

The respiratory signal has a low peak-to-peak amplitude typically in the range of 4 to $12 \mathrm{~mm}$. Variations are hence small and can be tracked accurately only by utilizing highresolution pulses. The relationship between the range resolution $\delta r$ and the pulse width is given by:

$$
\delta r=\frac{T_{w} \cdot c}{8}
$$

where $T_{w}$ is the width of the pulse in the time domain and $c$ is the speed of the electromagnetic waves. The required Nyquist sampling rate is given by [12]:

$$
f_{N}=\frac{4}{T_{w}}
$$

Obtaining a range resolution of $2 \mathrm{~mm}$, for example, requires a pulse width of 50 picoseconds. This translates into a Nyquist sampling rate of $80 \mathrm{GHz}$, which is too high for any practical purpose. For signals which exhibit repetitive nature, Equivalent Time Sampling (ETS) $[13,14,15]$ provides a solution by sampling at progressively increasing time intervals. This progressive sampling approach, however, adds complexity to the design of the sampler. In Section 3.2, we build upon the idea of ETS and present a novel scheme that enables us to recover UWB signals while operating at sub-Nyquist rates. Compared with the ETS approach, our scheme utilizes uniform sampling and rely on a simple constrain to guarantee signal recovery while sampling at sub-Nyquist rates and thereby avoiding any design complications. 


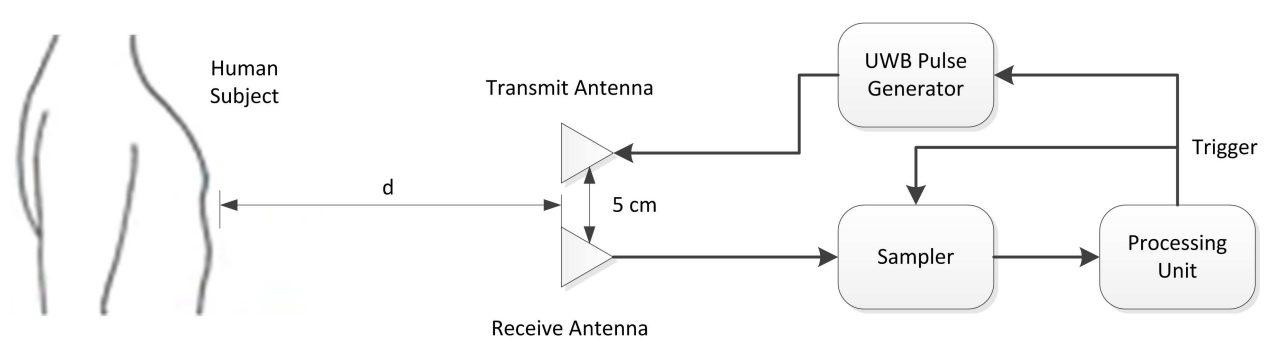

Figure 2: Illustration of the positioning of the transreceiver in relation to the human subject

\subsection{Multipaths}

The respiratory motion of a human consists of the movement of the chest wall and the abdomen. The chest expands or shrinks as air is taken in and out of the lungs. To monitor the respiratory movements, a UWB transreceiver directed towards the chest can be used as shown in Figure 2. The transmitter emits pulses that are reflected off the chest of the human subject. However, due to multipaths, these reflections will be superimposed by the reflections of the same pulses off other objects in the environment, creating an impulse response that is expected to be non-sparse in general. The components of the impulse response reflected off objects other than the chest of the human subject, called the background, can be more dominant than the components reflected off the chest, which is our signal of interest. It is therefore essential to eliminate the background before useful information can be extracted from the measurements. In the case where the background is assumed known or constant, it can be estimated by averaging measurements over a certain period and subtracting the average from subsequent measurements [17]. However, in practical situations, the background might be unknown and time varying, which suggests that a more robust background removal scheme is needed. In Section 3.2, we propose a novel technique for mitigating the background effect.

\section{Signal Model}

We approach our problem from a sparse signal estimation perspective, which allows us to exploit the sparse nature of the UWB impulse response to obtain high performance at low SNR values. The novel technique proposed here utilizes a modified form of the multiple 
pulse transmission scheme, which helps to reduce the required sampling rate. We first revisit the typically used multi-pulse signal model utilized for improving the SNR in Section 3.1 and then we modify this model in Section 3.2. We present the expressions for the single path case which is sufficient for our formulation due to our scheme for background removal presented in Section 3.3.

\subsection{A Multi-Pulse Transmission Scheme for Improved Signal-to-Noise Ratio}

In typical multi-pulse transmission schemes [5], the transmitted signal consists of a sequence of $N$ pulses as shown in Figure 3 and expressed mathematically as

$$
s(t)=\sum_{k=0}^{N-1} p_{t x}\left(t-k T_{p}\right) .
$$

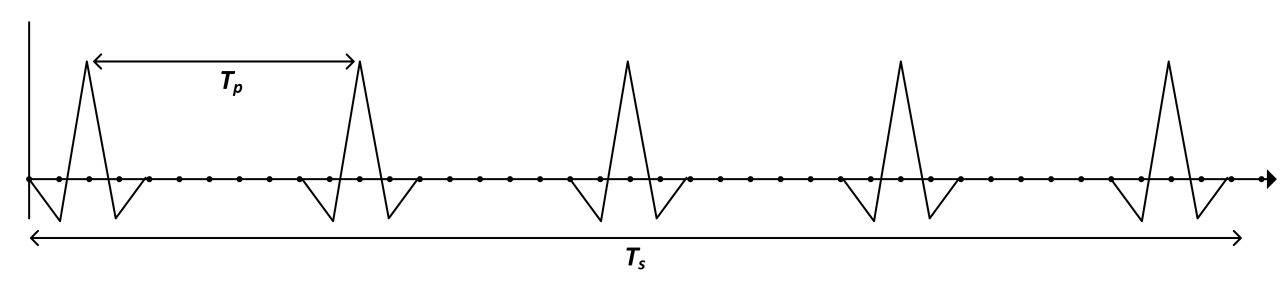

Figure 3: The transmitted pulse sequence for SNR improvement

All pulses have a duration of time $T_{w}$ and are separated by intervals of duration $T_{p}$. These pulses are transmitted within a single measurement window of time period $T_{s}$. Here, $T_{p}$ is assumed to be greater than $T_{w}$ and chosen such that the multipaths die out before transmission of the next pulse in the sequence. The transmitted pulse, $p_{t x}$, used here is the second derivative Gaussian pulse as given in (1).

The received pulses will be delayed versions of the transmitted pulses by time $\tau$, which is incurred due to the round-trip distance between the transreceiver and the reflecting object. The overall measurement is the average of the reflections over all such transmitted pulses within the $T_{s}$ time window and is given by the expression

$$
y(t)=\frac{1}{N} \sum_{k=0}^{N-1}\left(g\left(t-k T_{p}-\tau\right)+n(t)\right),
$$


where $g(t)$ is the reflected signal and $n(t)$ is zero-mean white Gaussian noise with variance $\sigma_{n}^{2}$.

The general measurement model from the use of such scheme can be expressed using vector-matrix notation as

$$
\mathbf{y}=\mathbf{A h}+\mathbf{n},
$$

where $\mathbf{y}$ is the $m \times 1$ measurement vector, $\mathbf{A}$ is the measurement matrix of size $m \times n$ and $\mathbf{n}$ is a vector of size $m \times 1$ representing zero-mean white Gaussian noise. $\mathbf{h}$ is the sparse vector of size $n \times 1$ to be estimated and composed of two parts; $\mathbf{h}_{\mathbf{c}}$, representing the impulse response created by the subject's chest movement, and $\mathbf{h}_{\mathbf{b c}}$, representing the impulse response created by the background objects in the environment. The measurement matrix for the specific transmission scheme in (5) has a Toeplitz structure in which each column is a shifted version of the transmitted UWB pulse. We denote this by matrix $\overline{\mathbf{A}}$

$$
\overline{\mathbf{A}}=\left[\begin{array}{cccc}
p(0) & & & \\
p(1) & p(0) & & \\
\vdots & p(1) & \ddots & \\
p(k-1) & \vdots & \ddots & p(0) \\
& p(k-1) & & p(1) \\
& & \ddots & \vdots \\
& & & p(k-1)
\end{array}\right]
$$

where $[p(0), p(1), \ldots, p(k-1)]$ are the samples of the transmitted pulse at Nyquist rate.

\subsection{Multi-Pulse Transmission for Reduced Sampling Rate}

By modifying the scheme in Section 3.1, we have developed a novel technique that enables us to sample the received signal at sub-Nyquist frequencies, while still achieving the accuracy offered at the Nyquist rate. The technique is based on utilizing as many pulses in the transmit sequence as the intended amount of subsampling, i.e., $N$ pulses for subsampling by a factor of $N$ compared to the Nyquist rate. The received sequence is then the delayed version of the transmitted pulse sequence according to the distance of the reflecting ob- 
ject from the transreceiver. However, unlike the scheme in Section 3.1, where we perform averaging at the receiver over the received multiple pulses, here we simply subsample the received sequence by a factor of $N$. Under the condition that the number of samples in the pulse interval $T_{p}$, are equal to $m N-1$, where $m$ is any integer, we can guarantee the recovery of the same samples as when sampling a single pulse with the Nyquist rate, albeit with different permutation. This means that we are utilizing multiple pulses to recover the same information that would effectively be carried by a single pulse but in this way we relax the requirement on the sampling rate at the receiver side, which is the main bottleneck in any high-frequency system. The condition $L_{p}=m N-1$, where $L_{p}$ is the number of samples in the pulse interval $T_{p}$, is to ensure that the samples are taken from unique locations from each of the received multiple pulses and can easily be satisfied by slightly extending $T_{p}$. The transmission scheme remains the same as in Figure 3 with the addition of the new constraint and mathematical expression given by

$$
s(t)=\sum_{k=0}^{N-1} p_{t x}\left(t-k T_{p}\right) \quad \text { s.t. } \quad L_{p}=T_{p} f_{N}=m N-1 .
$$

The resulting measurement, $y(t)$, is constructed by concatenating the received sequence over all $N$ transmitted pulses and subsampling it by a factor of $N$. Mathematically, this can be expressed as

$$
y(t)=\left[\sum_{k=0}^{N-1}\left(g\left(t-k T_{p}-\tau\right)+n(t)\right)\right]_{\downarrow N},
$$

where $\downarrow N$ denotes subsampling by a factor of $N$ and the delay, $\tau$, is defined by the distance of the reflecting object from the transreceiver as before. Apart from the constraint given above, the amount of subsampling is only limited by the practical considerations of the problem i.e. the acceptable length of the measurement time window $T_{s}$. In general, $T_{s}$ should be sufficiently small such that the received signals at each pulse interval $T_{p}$ are identical.

The recovery of the pulse samples using the subsampling approach presented above is illustrated in Figure 4 for $N=5$. Here $L_{p}=9$ which satisfies the condition given above. The circled points are the sampling instances according to the subsampling ratio $N=5$ and 
it can be seen that all the required samples are recovered from the pulses in the received sequence. Similar to the model presented in Section 3.1, the signal model here can also be represented in the form of (6) with the difference in the formulation of the measurement matrix $\widetilde{\mathbf{A}}$ whose columns are now the subsampled and shifted versions of the transmitted pulse sequence in (7), i.e.

$$
a_{j}=\left[s\left(t-(j-1) \delta t_{s}\right)\right]_{\downarrow N}
$$

where $a_{j}$ is the $j$ th column of matrix the $\widetilde{\mathbf{A}}$, with $j=1,2, \ldots, n$ and $\delta t_{s}$ is the sampling interval defined by the Nyquist sampling rate for the transmitted pulses.

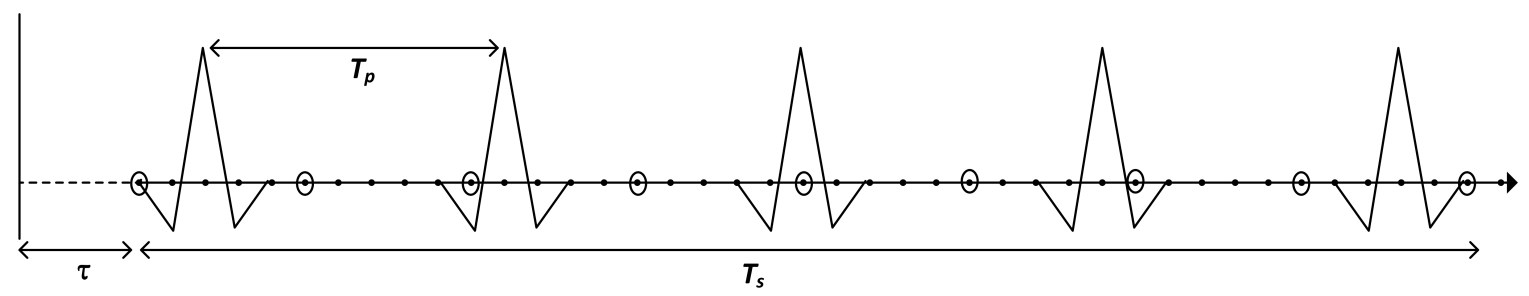

Figure 4: Illustration of pulse samples recovery by subsampling of the received sequence

\subsection{Multipath Effect and the Background}

The received signal, $y(t)$, comprises several multipath components of which only one component is assumed to be reflected off the chest of the human subject. This component of the measurement signal gives us information about the impulse response, $h_{c}(t)$, related to the subject's chest movements. All remaining multipath components correspond to the impulse response created by the objects other than the human subject in the environment. They are represented as $h_{b c}(t)$. Equation (6) can hence be written as

$$
\mathbf{y}=\mathbf{A}\left(\mathbf{h}_{\mathbf{c}}+\mathbf{h}_{\mathbf{b c}}\right)+\mathbf{n}
$$

The background component can be time variant but is assumed to remain constant over a short time duration such as the duration between two measurements. The impulse response, $h_{c}(t)$, varies in time as a result of being modulated by the subject's chest movements. Tracking variations in $h_{c}(t)$ will enable us to track variations in chest displacements.

Under the assumption that the background remains constant between any two consecu- 
tive measurements, we can eliminate this undesired signal component by using the difference between two consecutively received signals. The difference between the two measurements is expected to cancel out the effect of the multipath components, retaining only the signal of interest, $h_{c}(t)$, which would not cancel out due to the subject's chest movements ${ }^{1}$. The mathematical expression resulting from using equation (9) with $N=1$ is given by

$$
\mathbf{z}=\mathbf{y}_{\mathrm{t}-\delta \mathrm{t}_{\mathrm{b}}}-\mathbf{y}_{\mathrm{t}}=\mathbf{A}\left(\mathbf{h}_{\mathrm{t}-\delta \mathrm{t}_{\mathrm{b}}}-\mathbf{h}_{\mathrm{t}}\right)+\mathbf{n}_{\mathrm{t}-\delta \mathrm{t}_{\mathrm{b}}}-\mathbf{n}_{\mathrm{t}},
$$

where $\delta \mathrm{t}_{\mathrm{b}}$ represents the interval between any two consecutive measurements. Let

$$
\mathbf{n}_{\mathrm{t}-\delta \mathrm{t}_{\mathrm{b}}}-\mathbf{n}_{\mathrm{t}}=\mathbf{m}_{\mathrm{t}}
$$

then,

$$
\mathbf{z}_{\mathrm{t}}=\mathbf{A}\left(\mathbf{h}_{\mathbf{b c}\left(\mathrm{t}-\delta \mathrm{t}_{\mathrm{b}}\right)}+\mathbf{h}_{\mathbf{c}\left(\mathrm{t}-\delta \mathrm{t}_{\mathrm{b}}\right)}-\left\{\mathbf{h}_{\mathbf{b c}(\mathrm{t})}+\mathbf{h}_{\mathbf{c}(\mathrm{t})}\right\}\right)+\mathbf{m}_{\mathrm{t}} .
$$

For $\delta \mathrm{t}_{\mathrm{b}}<1$ second, $\mathbf{h}_{\mathbf{b c}\left(\mathrm{t}-\delta \mathrm{t}_{\mathrm{b}}\right)}=\mathbf{h}_{\mathbf{b c}(\mathrm{t})}$ and hence

$$
\mathbf{z}_{\mathrm{t}}=\mathbf{A} \mathbf{h}_{\mathbf{d}(\mathrm{t})}+\mathbf{m}_{\mathrm{t}},
$$

with $\mathbf{h}_{\mathbf{d}(\mathrm{t})}=\mathbf{h}_{\mathbf{c}\left(\mathrm{t}-\delta \mathrm{t}_{\mathrm{b}}\right)}-\mathbf{h}_{\mathbf{c}(\mathrm{t})}$; i.e., the differential impulse response.

\section{The SABMP Algorithm}

Sparsity implies that the vector to be estimated is expected to have only a few non-zero elements. After the removal of the multipath effects, the remaining impulse response, $\mathbf{h}_{\mathbf{d}(\mathbf{t})}$, is a sparse vector with the locations of nonzero elements dependent on the current location of the chest. Several greedy algorithms have been developed for sparse signal estimation. Algorithms like Orthogonal Matching Pursuit (OMP) [18] operate based only on the sparsity information without considering any signal or noise characteristics. Other algorithms, like

\footnotetext{
${ }^{1}$ We expect the background to remain constant most of the time and hence the assumption $h_{b c}\left(t-\delta t_{b}\right)=$ $h_{b c}(t)$. At the specific instances when this assumption fails, the algorithm might fail to track the breathing pattern momentarily. However, as the background environment stabilizes, we again resume tracking. Note that this brief loss of tracking is by itself useful information as it gives information about the movement of the patient in his/her sleep which is an important parameter in sleep apnea studies.
} 
Fast Bayesian Matching Pursuit (FBMP) [19] and SABMP [21], use the Bayesian statistics of the signal and noise, along with sparsity rate, to compute the sparse vector estimate.

SABMP has the advantage that it makes no assumption on the distribution of the signal to be estimated, which makes it suitable for applications where signal statistics might be unknown [20]. The noise is still assumed to be Gaussian, which is a reasonable assumption in most cases. The initial estimate of sparsity provided to the algorithm is refined after each greedy search for the estimation of the sparse vector.

The MMSE estimate in the SABMP algorithm is given by the expression

$$
\hat{\mathbf{h}}_{m m s e} \triangleq E[\mathbf{h} \mid \mathbf{y}]=\sum_{S} p(S \mid \mathbf{y}) E[\mathbf{h} \mid \mathbf{y}, S]
$$

where $\mathbf{h}$ is the sparse vector to be estimated with unknown distribution of the non-zero elements and $\mathbf{y}$ is the set of available observations. The sum is over all possible $2^{n}$ supports $S$, where $n$ is the dimension of $\mathbf{h}$. This means that the SABMP algorithm explores the entire dimension, $n$, of the vector to be estimated to provide the MMSE solution. Moreover, the SABMP algorithm outperforms most other algorithms in speed and estimation accuracy.

The main input arguments to the algorithm in our case are the differential measurement vector, $\mathbf{z}$, the measurement matrix, $\mathbf{A}$, a parameter, $r_{\text {stop }}$, which defines the refinement limit, and an initial estimate of the sparsity rate, $p$. Information on the remaining algorithmic parameters and detailed derivations can be found in [20,21].

\section{Respiratory Movement Tracking Algorithm}

The signal model from subsections 3.2 and 3.3 provides us with means for obtaining the round-trip delay information for the UWB pulses interacting with the chest of the human subject. After background removal, the differential impulse response, $h_{d}(t)$, consists of a single cluster of non-zero taps and can be modeled as a sparse vector. The locations of these non-zero elements within the sparse vector carry information on the round-trip delay, which is dependent on the current distance of the chest from the ultra-wideband transreceiver. The respiratory movements induce a variable path delay for the reflected pulse and hence the 
location of these non-zero elements moves up and down periodically under our transreceiver setup shown in Figure 2. By estimating this sparse vector and the locations of the non-zero elements (or more specifically that of the largest element) in it, we can track the movement of the chest and estimate the respiratory signal. We then employ the SABMP algorithm for estimating the sparse vector, $h_{d}(t)$, and obtain the delay information using the expression

$$
\tau=\underset{i}{\operatorname{argmax}}\left(\mathbf{h}_{\mathbf{d}}[i]\right),
$$

where $i$ is the index of the vector locations from $1: n$. Note that although the differential impulse response $h_{d}(t)$ also contains chest impulse response components from the previous time instance, they do not effect the estimation of $\tau$ through equation (14) because of their negative amplitudes.

The time delay information is then used to obtain the current distance of the chest wall from the transreceiver using

$$
d=\frac{\tau \cdot c}{2 f_{N}} .
$$

We note that here we used the sampling frequency defined according to the Nyquist criterion, $f_{N}$, and not the sub-Nyquist frequency, $f_{S}$, at which the received signal is actually sampled.

The proposed scheme is summarized below for convenience:

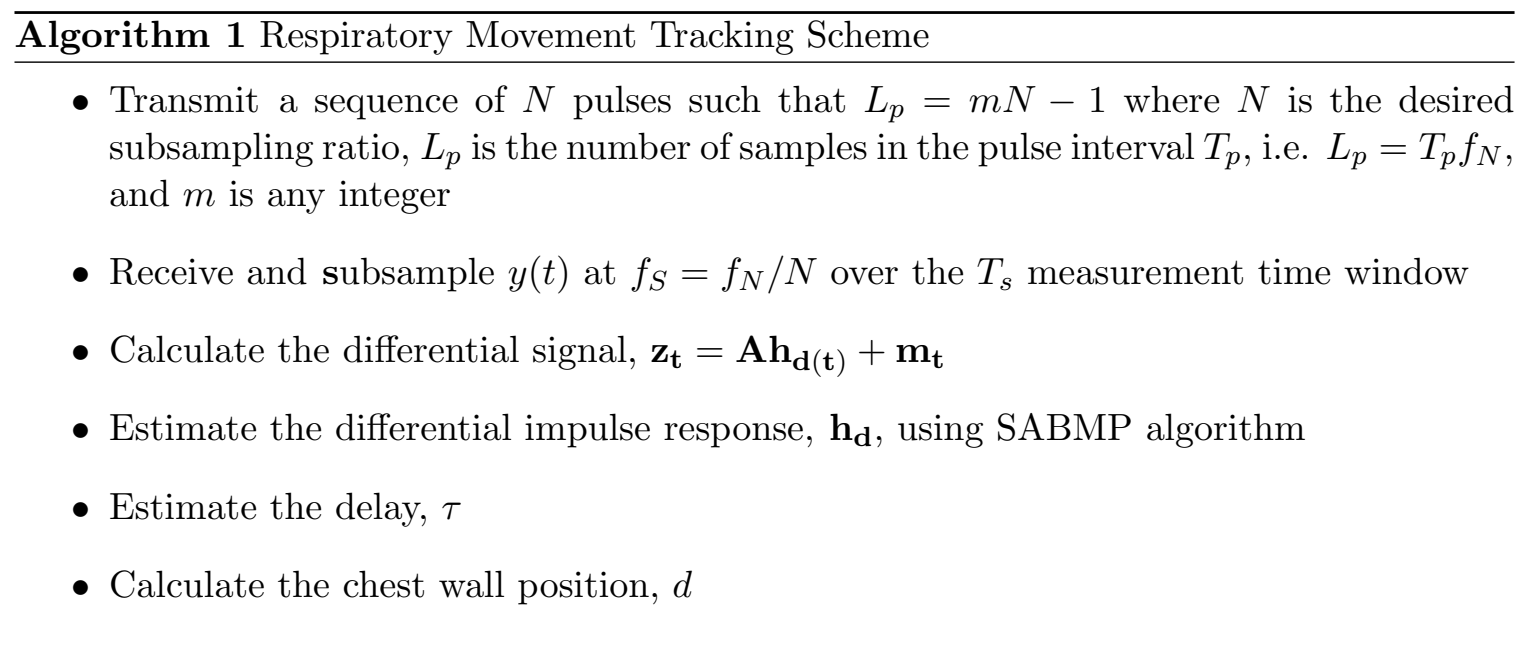




\section{Simulations}

Simulations were performed to test the performance of the proposed scheme using a prerecorded respiratory signal. The data utilized here is part of a larger dataset made publicly available by PhysioNet.org [22] and has been recorded using standard Polysomnography equipment for diagnosis of respiratory and sleep disorders. The recorded data represent normal respiratory movements of a human subject having a duration of 40 seconds. The recording was made while the subject was asleep. The signal is plotted in Figure 5 and was used to modulate the transmitted sequence to generate the synthetic measurements used in our simulations.

We used the IEEE 802.15.3a UWB channel model to generate the background that was added to each of the synthetic measurements. The qualitative performance of our proposed scheme can be analyzed by the quality of signal waveform reconstruction, which is useful for the physician to detect abnormal breathing patterns. The quantitative performance can be measured in terms of the root mean-square-error (RMSE) defined as

$$
R M S E=\sqrt{E\left\{(\hat{d}-d)^{2}\right\}},
$$

where $\hat{d}$ is the estimated and $d$ is the true position of the chest.

Figure 6 shows the tracking performance of the proposed scheme at the Nyquist sampling rate. The signal exhibits a peak-to-peak amplitude of approximatately $11 \mathrm{~mm}$ and in order to reconstruct the signal properly, we set our desired range resolution, $\delta r$, to $2 \mathrm{~mm}$, which is achieved using a pulse width, $T_{w}$, of 50 picoseconds with the associated Nyquist sampling rate being $80 \mathrm{GHz}$. The estimated signal shows good tracking performance, achieving a range resolution, $\delta r$, of $2 \mathrm{~mm}$ offered by the utilized pulse width. Higher resolutions can be also be obtained by using finer pulses, but for the purpose of qualitative analysis, this resolution is sufficient. As discussed in Section 2.2, sampling the received signal at the Nyquist rate can be computationally expensive. However, with the subsampling scheme of Section 3.2, we are able to achieve the same Nyquist rate performance while sampling at a much lower rate of $f_{S}=2.1 \mathrm{GHz}$ using $N=37$ in the transmit sequence of equation (7) and chosen according to the given constraint. Figure 7 shows the tracking performance for 
the subsampled case, which is very similar to the one in Figure 6.

Next, to evaluate the performance of our background removal technique, we test our proposed scheme with a slowly changing background environment. Figure 8 shows the collection of measurement vectors, $\mathbf{y}$, over time where the background environment changes slowly between time $t=21$ to $t=24$ seconds. The tracking performance of the algorithm is shown in Figure 9 where it can be seen that the algorithm loses track of the respiratory movements for a short duration around the time of change in the background environment but then it is able to regain tracking as soon as the environment stabilizes.

We also tested our proposed scheme with a background environment that starts changing abruptly at time $t=21$ seconds before stabilizing after 10 measurement cycles to a very different background profile at $t=22$ seconds. Figure 10 shows the collection of measurement vectors, $\mathbf{y}$, over time with the tracking performance of the algorithm shown in Figure 11 where it can be seen that the performance is similar to the previous case.

Finally, a quantitative analysis of the proposed scheme is presented. Figure 12 compares the tracking performance in terms of RMSE for two subsampling cases compared with that of the Nyquist rate sampling. Performance for the subsampled cases is slighty worse than the Nyquist rate at low SNR values but it can be seen that our proposed scheme performs well with SNR values of $10 \mathrm{~dB}$ or above with an RMSE of just around $0.1 \mathrm{~mm}$. As such, our subsampling approach has neglible effect on the tracking accuracy and does not degrade with increasing subsampling ratios particularly at good SNR levels. As mentioned earlier, the amount of subsampling is limited only by the constrained given in (7) and by the fact that $T_{s}$ should be limited such that both the chest movements and change in environment within this single measurement time window are neglible.

To analyze the performance enhancement attributed to the sparse signal estimation approach of our scheme and that of the Bayesian framework of the SABMP algorithm, we compare it with the OMP and Least Squares (LS) methods. In Figures 13 and 14, we plot the RMSE for both the Nyquist and sub-Nyquist cases against SNR values in the range of 0 to $30 \mathrm{~dB}$. The enhanced performance from the SABMP algorithm, which exploits both the sparsity and the Bayesian estimation framework, shows its usefulness as it achieves the minimum RMSE value at a much lower SNR compared with both the LS and OMP 


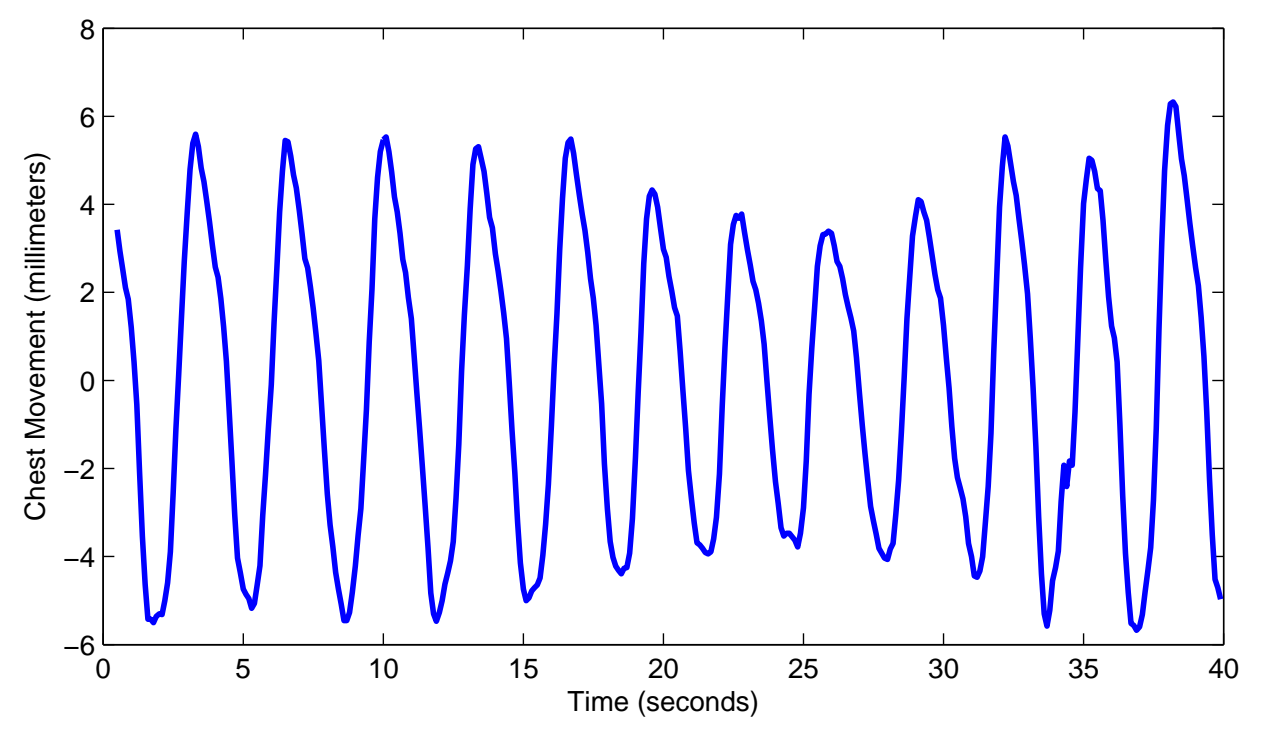

Figure 5: A pre-recorded respiratory signal

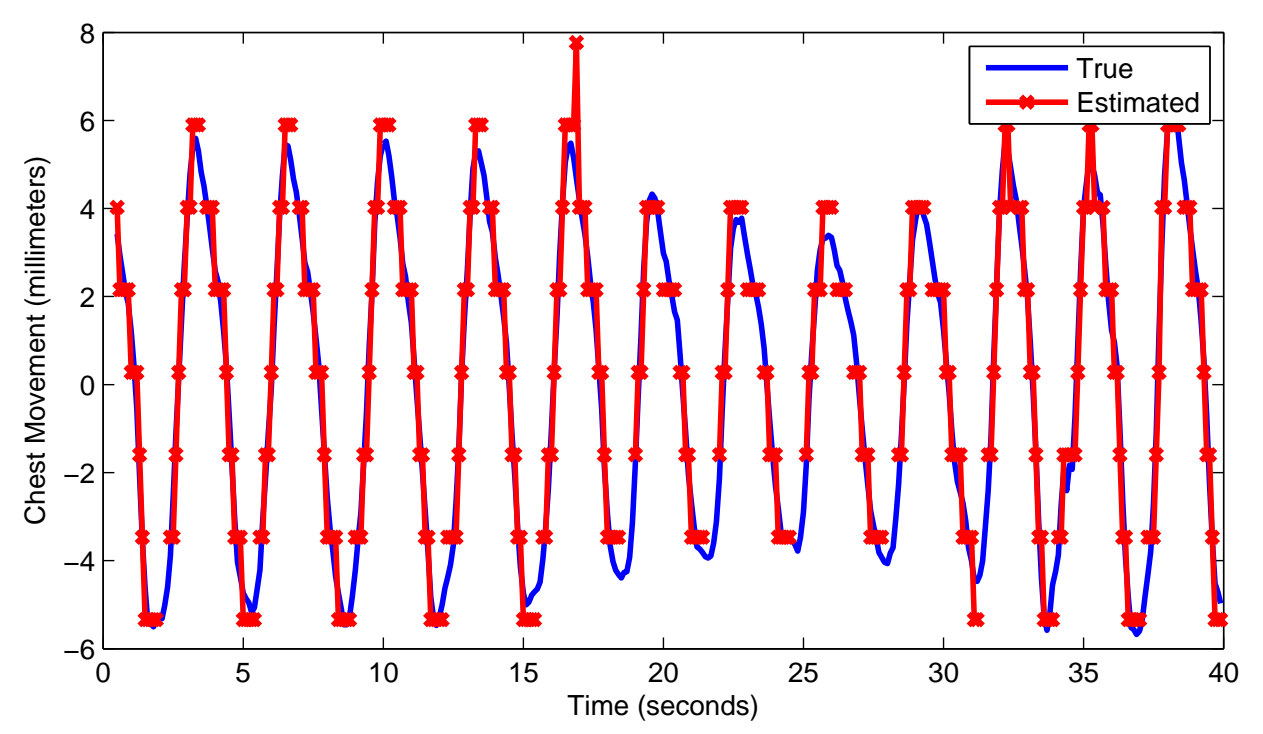

Figure 6: Signal reconstruction performance at $15 \mathrm{~dB}$ SNR with a Nyquist sampling rate of $80 \mathrm{GHz}$ 


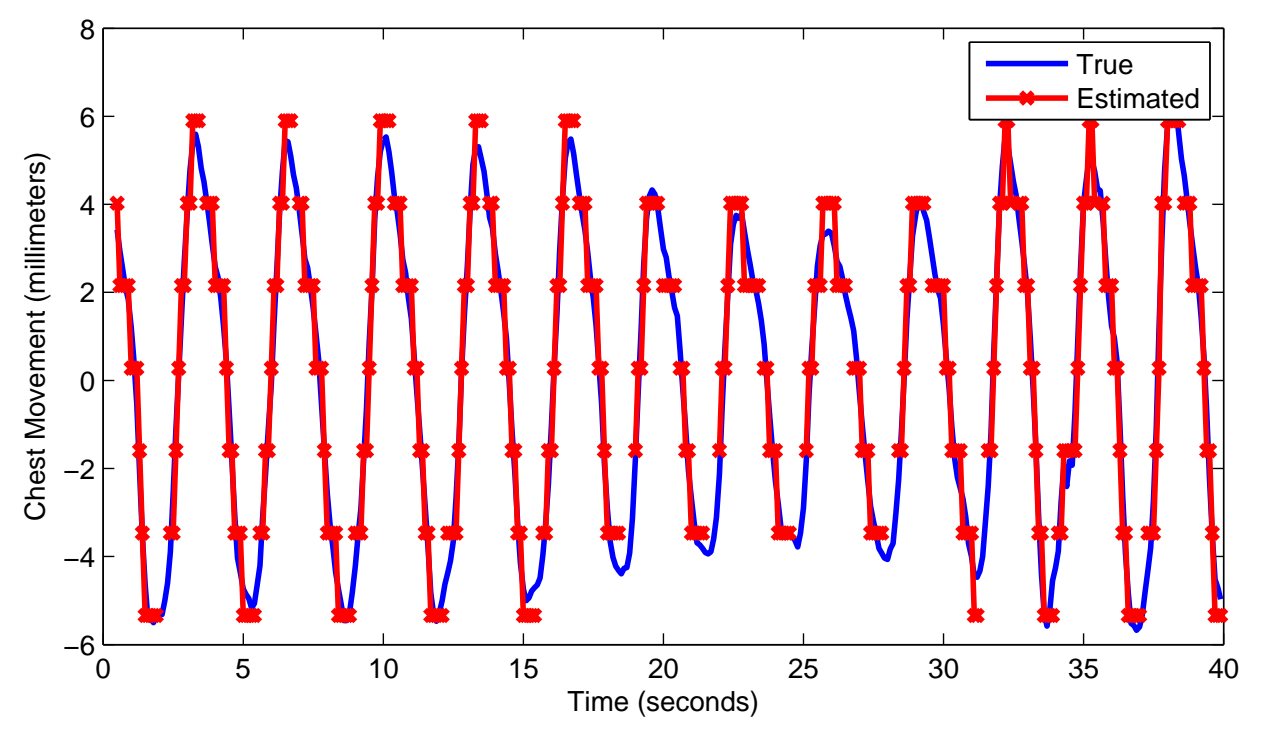

Figure 7: Signal reconstruction performance at $15 \mathrm{~dB}$ SNR with a sub-Nyquist sampling rate of $2.1 \mathrm{GHz}$

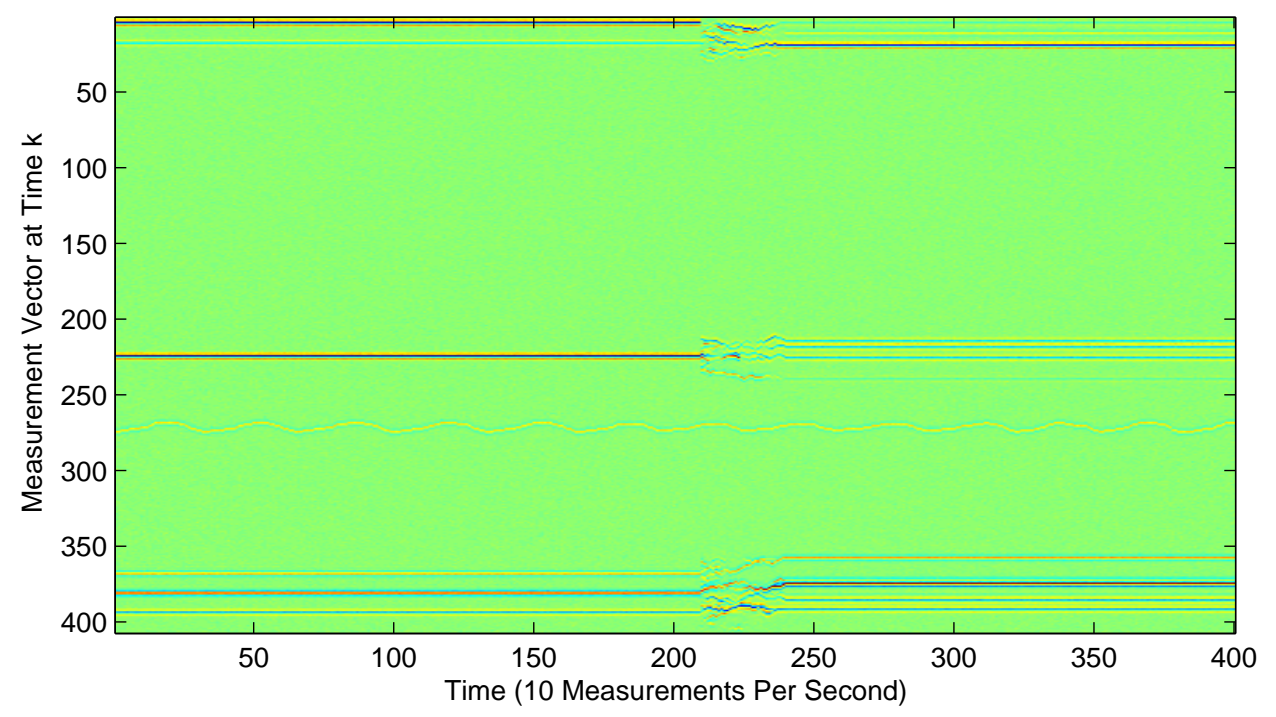

Figure 8: Measurement vectors in an environment with a slowly changing background 


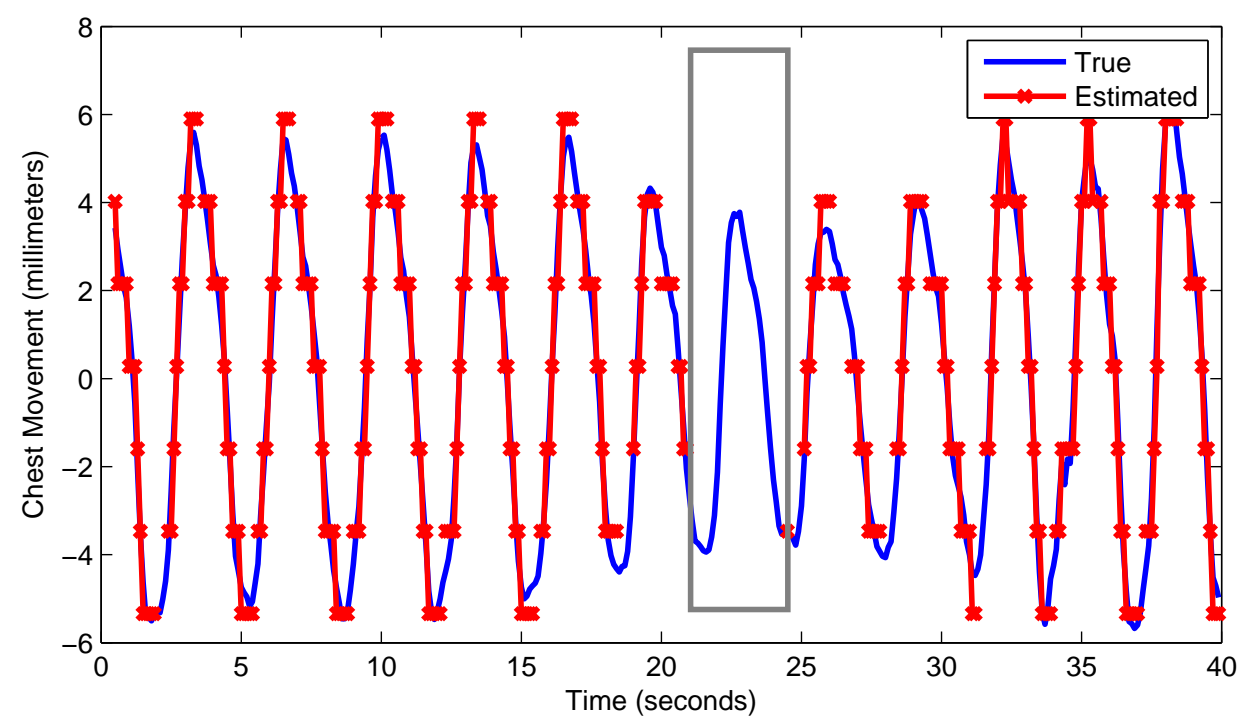

Figure 9: Tracking performance in an environment with a slow-varying background at 15 $\mathrm{dB}$ SNR and with a sub-Nyquist sampling rate of $2.1 \mathrm{GHz}$

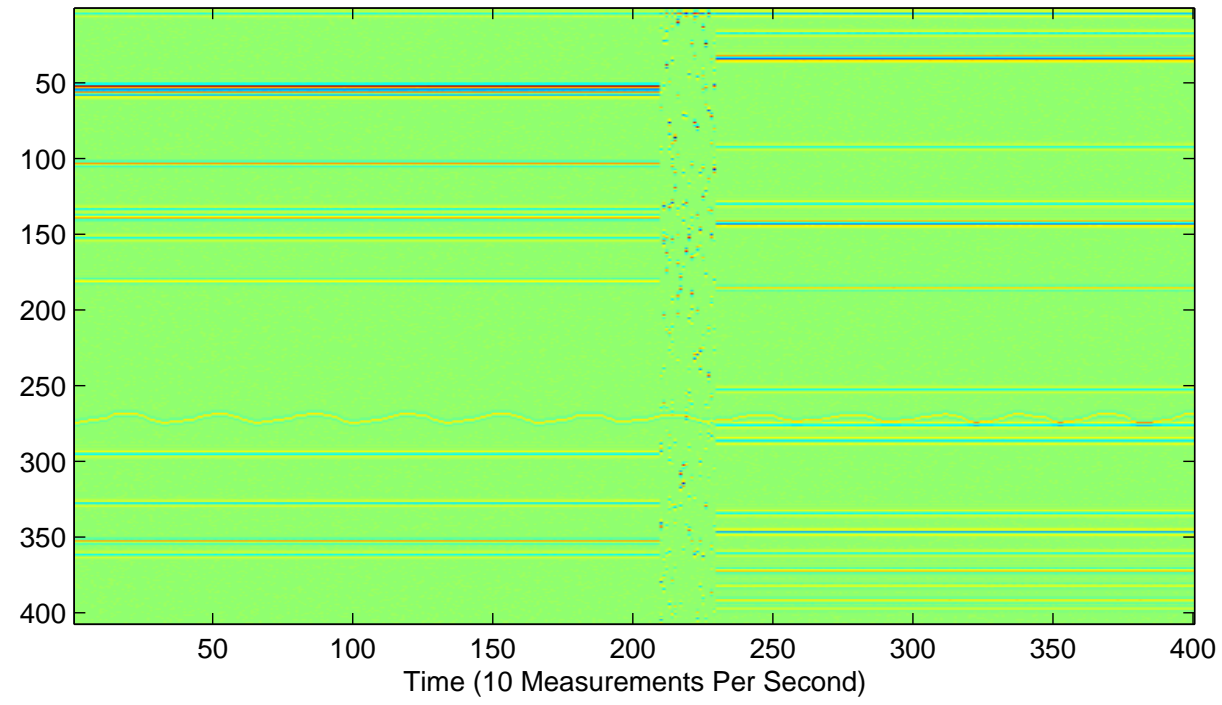

Figure 10: Measurement vectors in an environment with a fast changing background 


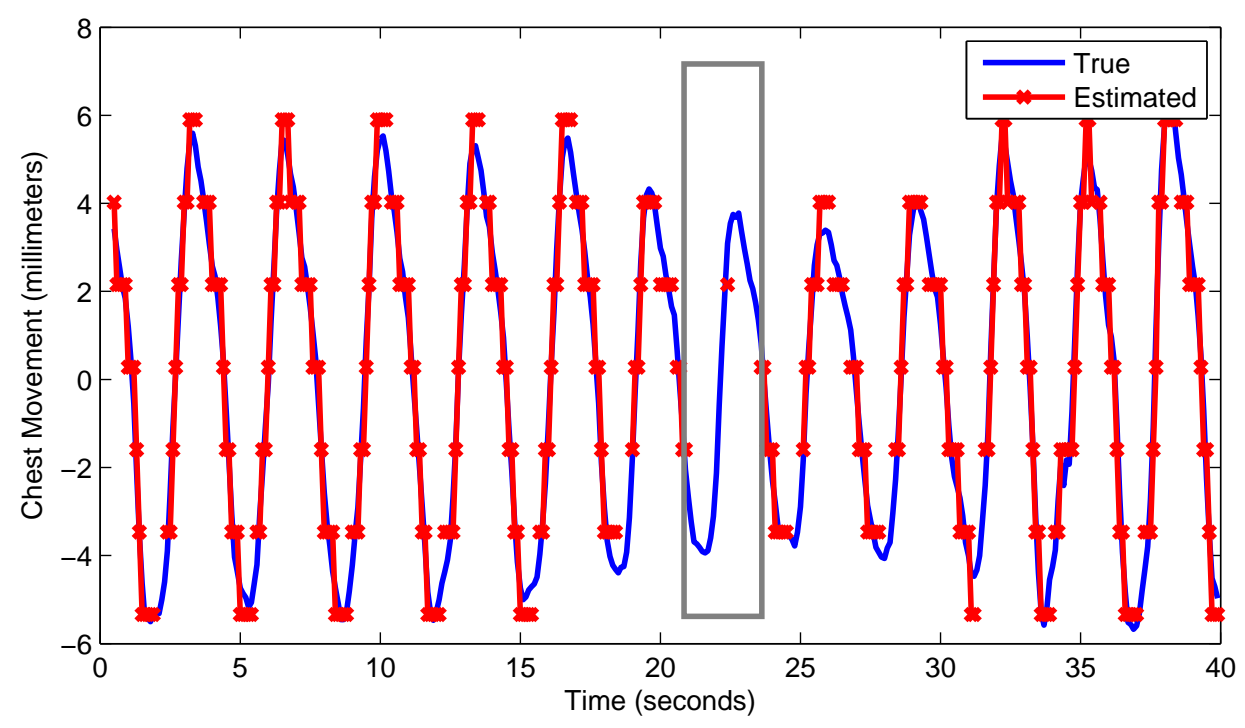

Figure 11: Tracking performance in an environment with a fast-varying background at 15 $\mathrm{dB}$ SNR and with a sub-Nyquist sampling rate of $2.1 \mathrm{GHz}$

methods.

\section{Conclusion}

Here, we presented a comprehensive scheme for tracking the respiratory movements of a human subject using ultra-wideband signals. We proposed techniques for efficient removal of background clutter and for significantly reducing the sampling rate of the system. Simulation results demonstrate that our tracking scheme is able to provide results that are as good as those possible with the Nyquist sampling rate. By exploiting the sparse nature of the respiratory movement tracking problem and using a Bayesian framework approach, our proposed scheme is able to provide accurate tracking performance at low SNR values. As such, our proposed scheme is capable of countering the challenges of low SNR, high sampling rates and multipaths and hence it provides a reliable low-complexity solution for the respiratory movement tracking problem. 


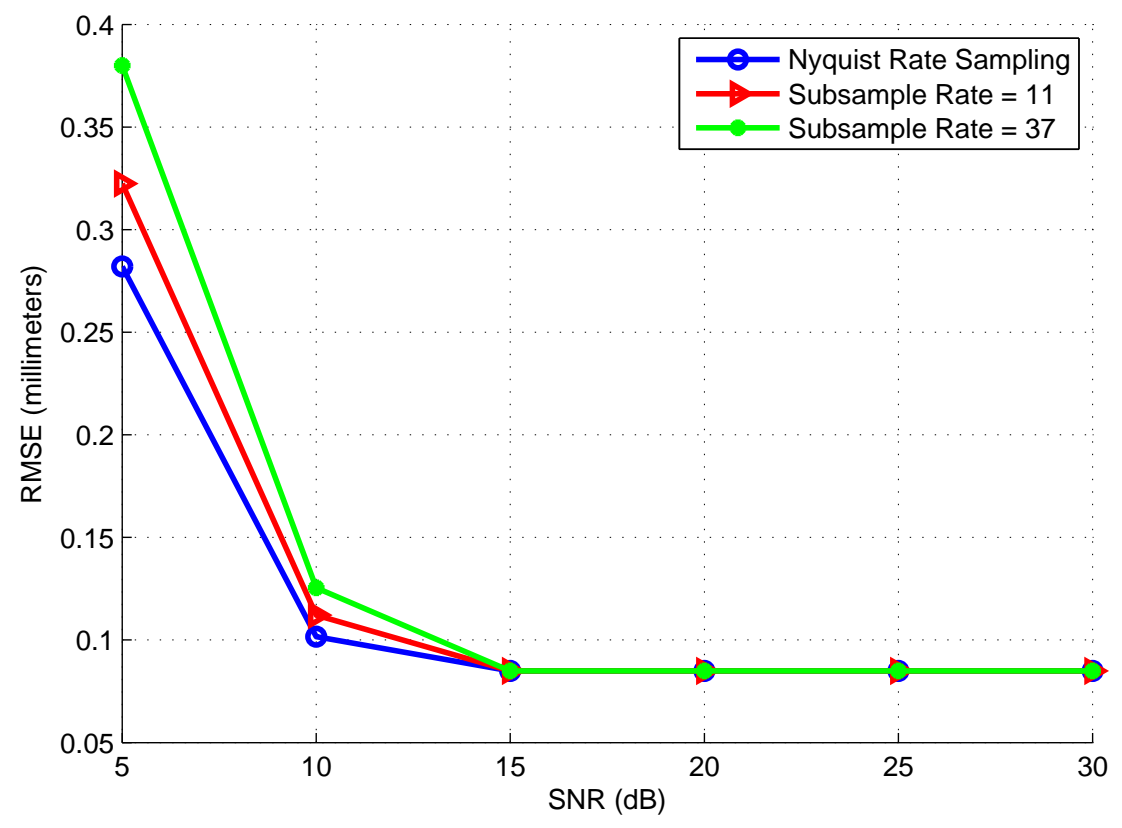

Figure 12: RMSE comparison between Nyquist and sub-Nyquist sampling rates

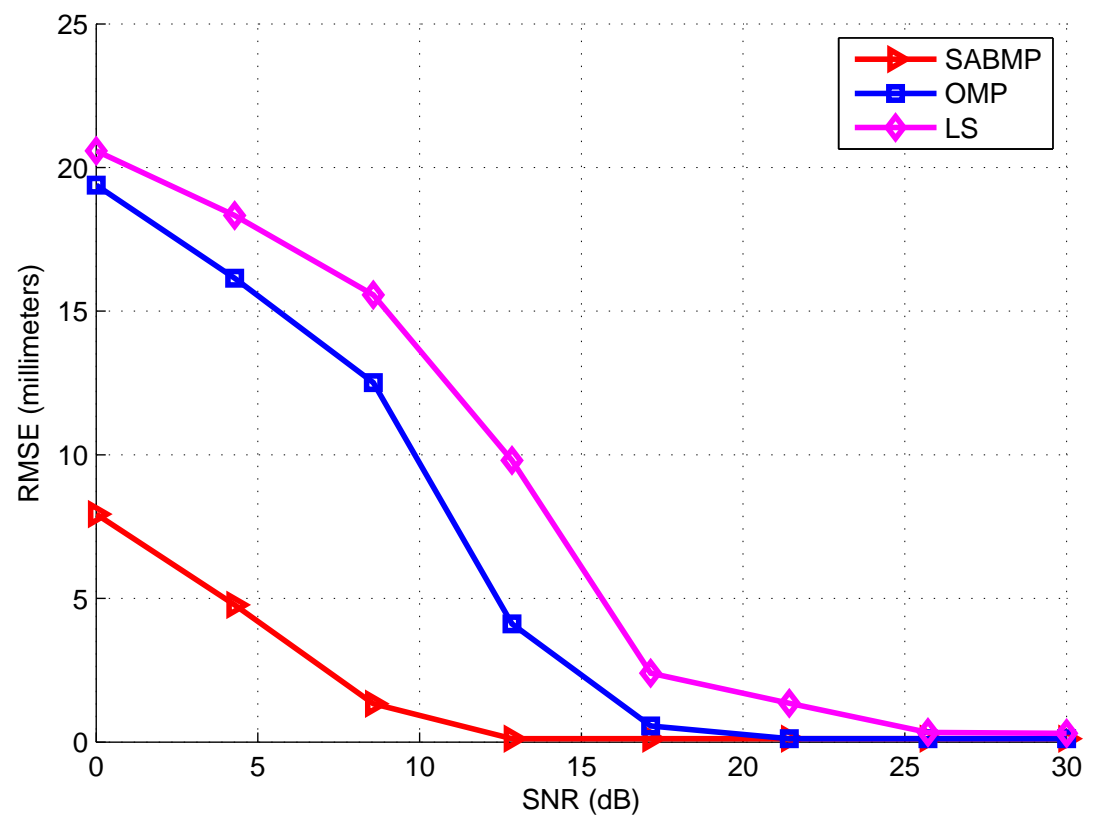

Figure 13: Performance comparison with non-sparsity based Least Squares (LS) and sparse non-Bayesian OMP algorithms at the Nyquist rate 


\section{References}

[1] National Heart, Lung and Blood Institute, U.S. Department of Health and Human Services; "What Is Sleep Apnea?".

[2] National Health Services, UK; "Diagnosing Sleep Apnoea".

[3] E.M. Staderini; "UWB Radars in Medicine", Aerospace and Electronic Systems Magazine, IEEE , vol.17, no.1, pp.13-18, Jan 2002.

[4] H. Soganci, S. Gezici, O. Arikan; "A Bayesian approach to respiration rate estimation via pulse-based ultra-wideband signals", Ultra-Wideband, 2009. ICUWB 2009. IEEE International Conference on , 9-11 Sept. 2009.

[5] S. Gezici; "Theoretical Limits for Estimation of Periodic Movements in Pulse-Based UWB Systems", Selected Topics in Signal Processing, IEEE Journal of , vol.1, no.3, pp.405-417, Oct. 2007.

[6] A. Lazaro, D. Girbau, R. Villarino, A. Ramos; "Vital signs monitoring using impulse based UWB signal", Microwave Conference (EuMC), 2011 41st European , 10-13 Oct. 2011.

[7] J.C.Y. Lai, Y. Xu, E. Gunawan, E.C. Chua, A. Maskooki, Y.L. Guan, K. Low, C.B. Soh, C. Poh; "Wireless Sensing of Human Respiratory Parameters by Low-Power Ultrawideband Impulse Radio Radar", Instrumentation and Measurement, IEEE Transactions on, vol.60, no.3, pp.928-938, March 2011.

[8] D. Dardari, A. Conti, U. Ferner, A. Giorgetti, M.Z. Win; "Ranging With Ultrawide Bandwidth Signals in Multipath Environments", Proceedings of the IEEE, vol.97, no.2, pp.404,426, Feb. 2009.

[9] Y. Nijsure, W. Tay, E. Gunawan, F. Wen, Y. Zhang, Y. Guan, A. Chua; "An Impulse Radio Ultra Wideband System for Contactless Non-invasive Respiratory Monitoring", Biomedical Engineering, IEEE Transactions on, vol.60, no.6, pp.1509,1517, June 2013. 
[10] S. Wu, Q. Zhang, R. Fan, N. Zhang, "Match-Filtering Based TOA Estimation for IR-UWB Ranging Systems", Wireless Communications and Mobile Computing Conference, 2008. IWCMC '08. International , 6-8 Aug. 2008

[11] X. Li, K. Pahlavan, J. Beneat; "Performance of TOA estimation techniques in indoor multipath channels", Personal, Indoor and Mobile Radio Communications, 2002. The 13th IEEE International Symposium on , vol.2, no., pp.911,915 vol.2, 15-18 Sept. 2002

[12] M.G. Di Benedett; "Ultra-wideband Communication Systems: A Comprehensive Overview:", EURASIP Book Series on Signal Processing and Communications, vol $5,2006$.

[13] R. A. Lawton, S. M. Riad, and J. R. Andrews; "Pulse and time-domain measurements," Proceedings of the IEEE, vol. 74, no. 1, pp. 77-81, 1986.

[14] K. Lawton and E. McConnell; "Equivalent Time Sampling for High-Speed Repetitive Signals Using E Series Boards and NI-DAQ Software", Application Note 066, National Instruments Corporation, 1995.

[15] E. Moreno-Garcia, J. M. De la Rosa-Vazquez, and O. Alonzo-Larraga; "An approach to the equivalent-time sampling technique for pulse transient measurements," in 16th International Conference on Electronics, Communications and Computers (CONIELECOMP 2006), 2006, pp. 34- 34 .

[16] L. Yang, G.B. Giannakis; "Ultra-wideband communications: an idea whose time has come”, Signal Processing Magazine, IEEE, vol.21, no.6, pp.26,54, Nov. 2004.

[17] G. Ossberger, T. Buchegger, E. Schimback, A. Stelzer, R. Weigel; "Non-invasive respiratory movement detection and monitoring of hidden humans using ultra wideband pulse radar", Ultra Wideband Systems, 2004. Joint with Conference on Ultrawideband Systems and Technologies. Joint UWBST \& IWUWBS. 2004 International Workshop on , 18-21 May 2004.

[18] Y.C. Pati, R. Rezaiifar, P. S. Krishnaprasad; "Orthogonal matching pursuit: recursive function approximation with applications to wavelet decomposition", Signals, Systems 
and Computers, 1993. 1993 Conference Record of The Twenty-Seventh Asilomar Conference on , 1-3 Nov 1993

[19] P. Schniter, L.C. Potter, J. Ziniel, "Fast bayesian matching pursuit", Information Theory and Applications Workshop, 2008 , Jan. 27 2008-Feb. 12008.

[20] Masood, Mudassir; Al-Naffouri, Tareq Y, "Sparse Reconstruction Using Distribution Agnostic Bayesian Matching Pursuit," IEEE Transactions on Signal Processing, November 1, 2013, Volume 61 Issue 21, pp 5298-5309.

[21] M. Masood and T.Y. Al-Naffouri, "Support Agnostic Bayesian Matching Pursuit for Block Sparse Signals", IEEE International Conference on Acoustics, Speech and Signal Processing (ICASSP 2013), Vancouver, Canada, May 2013.

[22] Goldberger AL, Amaral LAN, Glass L, Hausdorff JM, Ivanov PCh, Mark RG, Mietus JE, Moody GB, Peng CK, Stanley HE; "PhysioBank, PhysioToolkit, and PhysioNet: Components of a New Research Resource for Complex Physiologic Signals.", Circulation 101(23):e215-e220; 2000. 


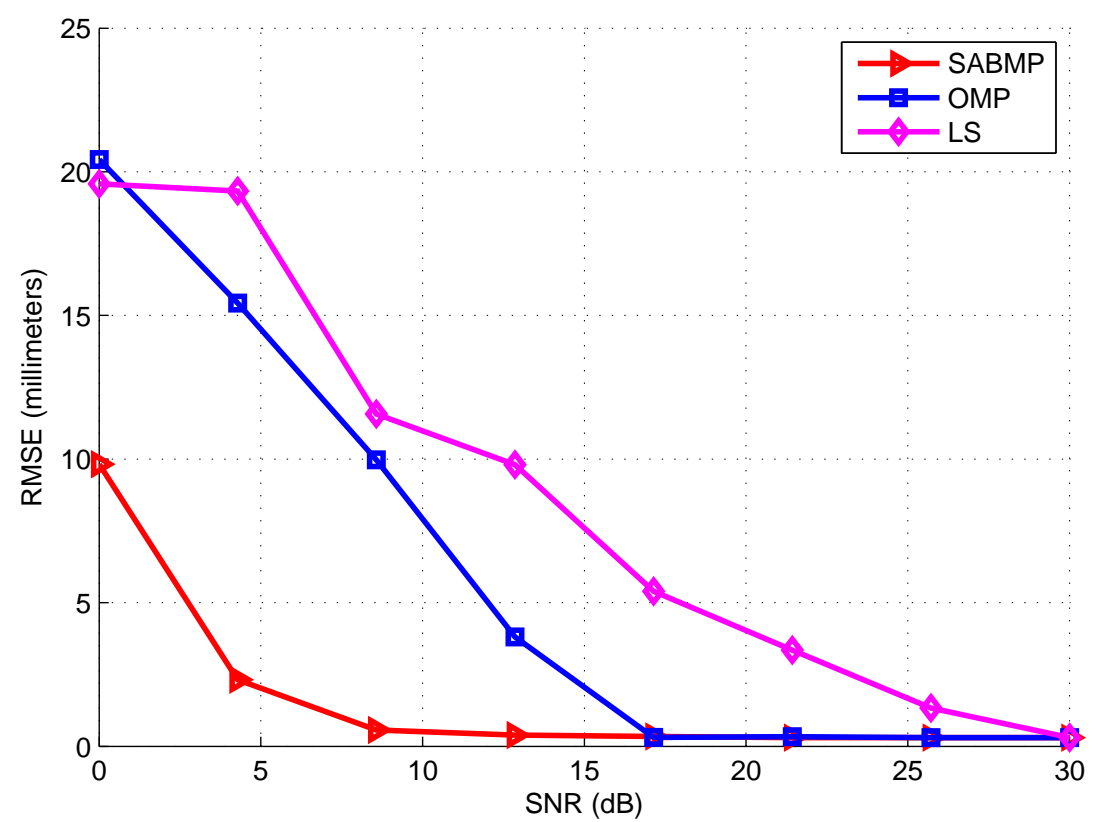

Figure 14: Performance comparison with non-sparsity based LS and sparse non-Bayesian OMP algorithms at the sub-Nyquist rate 